\section{ABCS}

\title{
Acknowledgements to reviewers
}

ABCS Health Sciences acknowledges the valuable contribution from scientific reviewers in 2019:

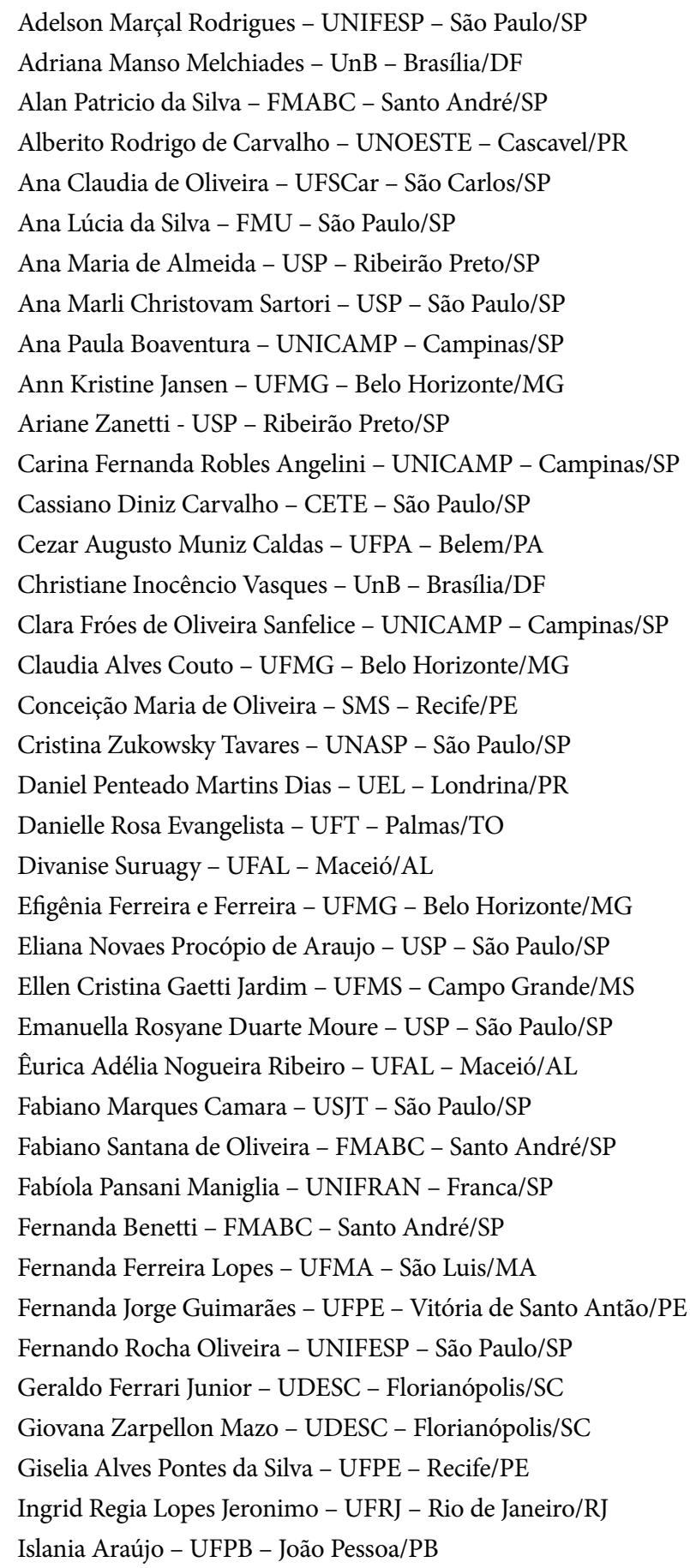
under the terms of the Creative Commons Attribution License

(C) 2020 ABCS Health Sciences 
Israel Gomy - UFPR - Curitiba/PR

Jeane Glaucia Tomazelli - INCA - Rio de Janeiro/RJ

Joice Anaize Tonon do Amaral - USP - São Paulo/SP

Jose Carlos Moleiro Junior - FMABC - Santo André/SP

Juliana Guimarães e Silva - FIOCRUZ - Rio de Janeiro/RJ

Karin Martins Gomes - UNESC - Crisciúma/SC

Karla Rona da Silva - UFMG - Belo Horizonte/MG

Kelli Cristina Silva de Oliveira - UNIFAFIBE - Bebedouro/SP

Lisie Alende Prates - UNIPAMPA - Uruguaiana/PA

Luciana Monteiro Vasconcelos Sardinha - MDS - Brasília/DF

Luciano Garcia Lourenção - FURG - Rio Grande/RS

Luis Fernando Barbosa Tavares - FMABC - Santo André/SP

Luís Flavio Sapori - PUC - Belo Horizonte/MG

Luiz Fernando Gouvêa e Silva - UFJ - Jataí/GO

Márcia Cristina Sangean - USP - São Paulo/SP

Marcia Fortes Wagner - IMED - Passo Fundo/RS

Marcia Maria Hernandes de Abreu de Oliveira Salgueiro - UNASP

- São Paulo/SP

Maria Auxiliadora Monteiro Villar - FIOCRUZ - Rio de Janeiro/RJ

Maria de Fatima Meinberg Cheade - UFMS - Campo Grande/MS

Maria Eliana Madalozzo Schieferdecker - UFPR - Curitiba/PR

Maria Goretti Pessoa de Araújo Burgos - UFPE - Recife/PE

Maria Ines Rosselli Puccia - FMABC - Santo André/SP

Marianne Oliveira Falco - UFG - Goiânia/GO

Marks Passos Santos - AGES - Jacobina/BA

Mauricio Braz Zanolli - FAMEMA - Marília/SP

Milva Maria Figueiredo de Martino - UNICAMP - Campinas/SP

Naerton José Xavier Isidoro - URCA - Crato/CE

Nancy Julieta Inocente - UNITAU - Taubaté/SP

Oscar Ribeiro - UA - Aveiro/Portugal
Paola de Oliveira Camargo - UFPel - Pelotas/RS

Patrícia Fernanda Roesler Bertolini - PUC - Campinas/SP

Patrícia Martins Bock - HCPA - Porto Alegre/RS

Patrícia Merly Martinelli - UNINORTE - Rio Branco/AC

Paulo Roberto Corsi - FCMSCSP - São Paulo/SP

Paulo Sérgio da Silva Santos - USP - Bauru/SP

Phillip Vilanova Ilha - UNIPAMPA - Uruguaiana/RS

Rafaela Catherine da Silva Cunha de Medeiros - UFRN - Natal/RN

Raquel de Deus Mendonça - UFOP - Ouro Preto/MG

Renata Cunha da Silva - UFPel - Pelotas/RS

Renata Miranda de Sousa - UFF - Niteroi/RJ

Renata Pimentel - UNESP - São Paulo/SP

Rita de Cássia Fernandes Grassia - HIAE - São Paulo/SP

Roberto Carlos Lyra da Silva - UNIRIO - Rio de Janeiro/RJ

Roberto José de Carvalho Filho - UNIFESP - São Paulo/SP

Rodrigo Wiltgen Ferreira - UFPel - Pelotas/RS

Rosélia Bezerra Paparelli - UNIP - São Paulo/SP

Rosely Erlach Goldman - UNIFESP - São Paulo/SP

Rosilene Andrade Silva Rodrigues - SES - Cuiabá/MT

Selma Denis Squassoni - FMABC - Santo André/SP

Stela Maris de Mello Padoin - UFSM - Santa Maria/RS

Sueli Coelho da Silva Carneiro - UERJ - Rio de Janeiro/RJ

Suhaila Mahmoud Smaili Santos - UEL - Londrina/PR

Tânia Cristina de Oliveira Valente - UNIRIO - Rio de Janeiro/RJ

Tenilson Amaral Oliveira - HMLMB - São Paulo/SP

Thiago Fernandes Pinto - USP - São Paulo/SP

Vanessa Comasseto Oliveira - UFPR - Curitiba/PR

Vera Catarina Castiglia Portela - UFRGS - Porto Alegre/RS

Viviane de Souza Pinho Costa - UNOPAR - Londrina/PR

Ymiracy Nascimento de Souza Polak - UFRJ - Rio de Janeiro/RJ 